\title{
Associations between high intensity zones, Modic and endplate changes in lumbar spine of low back pain patients
}

Kerstin Lagerstrand ( $\sim$ kerstin.lagerstrand@vgregion.se)

Goteborgs universitet Institutionen for kliniska vetenskaper

Helena Brisby

Goteborgs universitet Institutionen for kliniska vetenskaper

Hanna Hebelka

Goteborgs universitet Institutionen for kliniska vetenskaper

Research article

Keywords: Inter vertebral disc, endplate, vertebrae, Modic changes, high intensity zones, T2-mapping, low back pain, MRI, HIZ, LBP

Posted Date: November 1st, 2019

DOI: https://doi.org/10.21203/rs.2.16701/v1

License: (c) (i) This work is licensed under a Creative Commons Attribution 4.0 International License. Read Full License 


\section{Abstract}

Background High intensity zones (HIZ), Modic and endplate changes have all been pointed out as potential markers of low back pain (LBP). If an association between these morphological features exist, it may not only deepen the understanding of the underlying patho-physiological mechanism of LBP but may also improve the diagnostics by enabling stratification between individuals with non-specific LBP as well as within individuals having multi-segmental changes. The aim was to investigate if HIZ, Modic and endplate changes are associated and if endplate and vertebral T2-values reflect functional tissue characteristics related to these morphological features.

Methods 150 IVDs with corresponding endplates and vertebrae in 26 chronic LBP-patients (25-69y, mean 38y, 11 males) were examined with T1- and T2-weighted MRI, and T2-mapping. Associations between morphological features and between morphological features and functional T2-values were determined.

Results HIZ (62\% of patients, 1-2/patient) was associated with endplate changes ( $100 \%$ of patients, 1 $7 /$ patient) ( $p=0.0003$ and 0.0004 for upper and lower endplates), with an occurrence of $91 \%$ for upper and $71 \%$ for lower endplates adjacent to discs with HIZ. Modic changes (81\% of patients, $1-3 /$ patient) was associated with endplate changes $(p<0.0001)$ with an occurrence of $87 \%$ for endplates adjacent to vertebrae with Modic changes. The occurrence of both HIZ and Modic changes was $43 \%(p=0.0001)$ for upper and 29\% ( $p=0.003)$ for lower vertebrae. Significantly higher T2-values $(p<0.004)$ were found in the vertebral tissue with associated Modic changes and HIZ.

Conclusions This study of LBP-patients suggests that HIZ is associated with simultaneous presence of both Modic and endplate changes in the same motion segment. If these three simultaneous morphological features are linked to an active inflammatory process, reflected as a clinical specific pain profile remains to be investigated.

\section{Background}

Low back pain (LBP) is the most costly non-communicable endemic disease worldwide (1).The condition is multifactorial and in addition to central nervous system adaptations and responses, damage to the non-nervous spinal tissues is a key component. High intensity zones (HIZ), Modic changes (MCs) and endplate (EP) changes are known to be a part of the degenerative cascade and have been suggested to be associated with $\operatorname{LBP}(2,3)$. However, the association between such changes and LBP is not fully elucidated. Neither is their relationship with each other. In the search for reliable LBP-markers, better diagnostic tools are warranted $(4,5)$. The combination of HIZs, MCs and EP-changes could potentially be a stronger indicator of painful spine segments than the presence of these individual features alone. If such association exist, it may not only deepen the understanding of the underlying patho-physiological mechanism of LBP but may also enable stratification between individuals with non-specific LBP as well as within individuals having multi-segmental changes and thereby improve the diagnostics and clinical decision making. It has been suggested that $\mathrm{HIZ}$ is an effect of annular tears with accumulation of 
substances that are toxic to the disc cells and surrounding structures and linked with inflammation and degenerative changes $(6,7)$. Moreover, MC Type I, is likely of inflammatory origin, and associated with disruption and fissuring of the EPs $(8,9)$. Further, increased levels of pro-inflammatory mediators have been detected in the EP itself with adjacent subchondral bone edema (MC type I) when compared to EPs from vertebral fracture patients (10). Hence, such biochemical changes may compromise the function of the IVD, EP and vertebrae and play a crucial role in the development of LBP (11).

With the implementation of functional magnetic resonance imaging (MRI) methods, detection of early biochemical changes of the IVD, EP and vertebrae is feasible (12). Recent work has shown that in the presence of HIZs, altered IVD T2-values are detected at the position of the nucleus pulposus $(13,14)$. Even scarce in numbers, some T2-mapping studies have demonstrated subtle deterioration of the biochemical composition of the EPs $(15,16)$. Moreover, the feasibility of T2-mapping for objective characterization of the EPs and vertebrae has recently been demonstrated (17). This together with the fact that T2-mapping provide supplemental information about the tissue matrix and reflect presence of edema, makes the method a potential tool for monitoring of tissue changes linked to function. Finally, functional properties of the EPs may also be displayed using T2-mapping in combination with axial loading during MRI (alMRI) as compared to conventional imaging, when the spine is unloaded (UMRI) (17).

The aim of the study was firstly to investigate if HIZ, Modic and EP-changes are associated with each other and secondly to investigate if EP and vertebral T2-values reflecting functional tissue characteristics were related to these MRI findings.

\section{Methods}

\section{Study-cohort}

Twenty-six patients with chronic LBP (25-69y, mean 38y, 11 males), referred to the radiology department with non-specific LBP, were consecutively included. Inclusion criteria were severe LBP without radiating pain for more than 6 months, clinically severe enough to be considered for surgery, without signs of nerve root compromise during clinical examination, and age between 20-70 years. Exclusion criteria were previous spine surgery and contraindications for MRI.

\section{MRI}

The IVDs, EPs and vertebrae were examined (from superior EP L1 to vertebra S1) using a 1.5 T scanner (Magnetom Aera, Siemens Erlangen, Germany) with both the posterior and anterior phased array coil applied for increased signal-to-noise-ratio.

Standardized sagittal T1 weighted (T1W) MRI (320x320 matrix, slice thickness: $3.5 \mathrm{~mm}$, slice gap [SLG]: $0.7 \mathrm{~mm}$, field of view [FOV]: $300 \times 300 \mathrm{~mm}^{2}$, number of excitations [NEX]: 2) and standardized sagittal 
T2W-MRI (384x384 matrix, SLT: $3.5 \mathrm{~mm}$, SLG: $0.7 \mathrm{~mm}$, FOV 300×300 mm², NEX: 1), and axial T2W-MRI (256x256 matrix, SLT: $3.5 \mathrm{~mm}$, SLG: $0.7 \mathrm{~mm}$, FOV: 220x220 mm², NEX: 1) were performed. Additionally, all subjects were scanned with quantitative T2-mapping (256x256 matrix, SLT: $3.5 \mathrm{~mm}$, SLG: $0.7 \mathrm{~mm}$, FOV: 220x220 mm², NEX: 1). The T2-mapping of the spine was performed in the sagittal view covering L1-S1.

The participants were examined twice with the present protocol, initially with uMRI and subsequently with alMRI. T2-mapping was performed at the end of the protocol, approximately 20 minutes after the first measurement. Hence, the spine had been loaded for 20 minutes before T2-mapping.

The alMRI measurements were performed with a DynaWell compression device (DynaWell diagnostics $A B$, Las Vegas, NV, USA) with load, corresponding to $50 \%$ of the body weight.

\section{Post-processing if the MR images}

All post processing of the images was performed with the dedicated analysis tool of the scanner (Syngio Via, Siemens Erlangen, Germany).

The conventional T1W- and T2W-images were used for classification of IVD degeneration into Pfirrmann grade according to recommendations (18). The grading was performed by a senior radiologist (15 years of experience) and was based on the uMRI examinations using all images in the image stack.

Conventional images were also used for identification of HIZs, MCs and EP-changes (Figure 1), where the MCs were classified into MC Type I, II and II according to Modic et al. (19). HIZs was defined as the presence of a high signal located in the posterior annulus fibrosus, visible only on the T2W-but not on the T1W-images (20). EP-change was defined as apparent visual inhomogeneity and discontinuity in signal in the EP-zone (Figure 1), identified in the conventional MR images and restricted to the EP. EPs with Schmorl's nodules were not included. Identification of the morphological features was performed by the senior radiologist.

Associations between HIZs, MCs and EP-changes within the same motion segment were determined. To determine associations for superior and inferior EPs separately, the vertebrae were divided horizontally into two equally large parts, hereafter called superior and inferior vertebrae.

Functional behaviors of the EPs and vertebrae in terms of T2 were retrieved from T2-maps acquired both with $\mathrm{UMRI}$ and aIMRI. The T2-maps were reconstructed from optimized fitting of the raw data and then reformatted into $10 \mathrm{~mm}$ non-overlapping slices, where the three central slices were used in the estimation, thus, covering $30 \mathrm{~mm}$ of the EP and vertebral width. For determination of EP T2-values, the T2-maps were manually segmented into regions of interest (ROIs) covering the EP-zone (17). In general, the EP ROI was positioned approximately one pixel away from the visible edge of the IVD and vertebral body. With this strategy, the EP-zone was assumed to include both bony and cartilage EP. The median T2-value within the ROI was calculated and used as a measure of the EP T2-value. Similarly, the vertebral T2-value was 
determined in the superior and inferior vertebrae as the median value within these regions. All segmentations were performed by a trained observer, supervised by a senior radiologist with 20 years of experience. The intra- and interobserver ICC for the vertebral T2-value were excellent (0.9-1.0), for the superior EPs: fair to good (0.5-0.6) and for the inferior EPs: good to excellent (0.6-0.8).

\section{Statistics}

All statistical analyses were performed using SAS Software version 9.4 (SAS Institute Inc., Cary, NC).

For comparison between groups and differences within groups, a mixed linear model adjusted for multiple observations within subjects was used. The comparisons were performed with correction for Pfirrmann grades. Results are presented as adjusted means (with $95 \%$ confidence intervals) together with p-values. All tests were two-tailed at 0.05 significance level. To test difference in variances between groups, the two-tailed F-test was used at 0.05 significance level.

Inter-rater agreement for the T2-measurements was displayed using intraclass correlation coefficients (ICC) with 95\% confidence intervals. ICC model 2 was used with single measurement to determine consistency in agreement. ICC-values $<0.4$ represent poor agreement, $0.4-0.75$ represent fair to good agreement and $>0.75$ indicate excellent reliability.

\section{Results}

HIZs were found in $16 \%$ of the IVDs (in $62 \%$ of the patients, $1-2 /$ patient) and EP-changes were found in $39 \%$ of the EPs ( $100 \%$ of the patients, $1-7 /$ patients). Corresponding values for MC of any type and MC Type I were $13 \%$ ( $81 \%$ of the patients, $1-3 /$ patient)) and $5 \%$ (27\% of the patients, $1-3 /$ patient).

The patient cohort displayed the following Pfirrmann grade distribution, Pfirrmann 1-5: 8, 50, 29, 13, 0\%

\section{Associations between HIZ, MC and EP-changes}

Associations between the investigated morphological features are presented in Table 1. The presence of HIZ was associated with the presence of EP-changes ( $p=0.0003$ and 0.0004 for superior and inferior EPs, respectively), with an occurrence of $91 \%$ for superior and $71 \%$ for inferior EPs adjacent to IVDs with HIZ. Also, presence of MC of any type was associated with presence of EP-changes $(p<0.0001)$ with an occurrence of $87 \%$ for EPs adjacent to vertebrae with MCs. Finally, the occurrence of both HIZ and MC in the same segment was $43 \%(p=0.0001)$ for superior and $29 \%(p=0.003)$ for inferior vertebrae. The association between MC Type I findings and HIZ and EP-changes are not presented due to limitation in power. 


\section{Vertebral T2-values}

Associations were found between the vertebral T2-value (at uMRI) and the investigated morphological features. The T2-value was significantly higher for vertebrae with presence of HIZ in adjacent IVDs in comparison with no HIZ (103 \pm 11 vs $99 \pm 9, p=0.001)$. This was also true for superior $(102 \pm 11$ vs $99 \pm 9$, $p=0.001)$ and inferior vertebrae $(106 \pm 12$ vs $100 \pm 9 ; p=0.0003)$. As expected, the vertebral T2-value was found to be significantly higher for presence of MC compared to no MC (all: $104 \pm 11$ vs $98 \pm 11 \mathrm{~ms}$, $p=0.003$; superior: $101 \pm 11$ vs $97 \pm 11 \mathrm{~ms}, p=0.03$; inferior: $101 \pm 11$ vs $99 \pm 12 \mathrm{~ms}, p=0.001$ ), and for MC Type I compared to no MC Type I (all: $104 \pm 11$ vs $98 \pm 11 \mathrm{~ms} ; p=0.003$; superior: $101 \pm 11$ vs $97 \pm 11 \mathrm{~ms}, p=0.04$; inferior: $111 \pm 11$ vs $99 \pm 12 \mathrm{~ms}, p=0.001)$. The vertebral T2-value was not associated with EP-changes $(p>0.6)$.

Also for alMRI-uMRI, the investigated morphological features were not found to be associated with the vertebral T2-value $(p>0.5)$.

\section{EP T2-values}

Regardless of type of morphological changes in the segment, similar EP T2-values (at uMRI) were detected $(38 \pm 15,39 \pm 15,37 \pm 15,35 \pm 10$, and $34 \pm 9$ ms for no morphological change, EP-change, HIZ, MC any type, and MC Type $\mathrm{l} ; \mathrm{p}>0.1)$.

However, the effect of aIMRI on the EP T2-value varied depending on type of morphological changes in the segment. For EPs with no morphological changes in the EPs or in the adjacent vertebra, nor any adjacent HIZ, a large spread in the T2-value was seen for alMRI-uMRI (SD=12ms). A narrower T2distribution was statistically verified for EPs with MC any type and MC Type I ( 6 and $5 \mathrm{~ms}: p<0.001)$, but not for HIZ and EP-changes (11 and 8ms: p>0.07).

\section{Discussion}

This study shows that the presence of $\mathrm{HIZ}$ is associated with MCs. Furthermore, $\mathrm{HIZ}$ was found to be associated with EP-changes, which in turn were found to be associated with MCs. Hence, simultaneous presence of these morphological features in the same motion segment was a common characteristic in this LBP cohort, supporting previous observations with crosstalk between inflammatory IVD and vertebral changes (21). Moreover, T2-mapping was found to objectively reflect EP and vertebral tissue changes associated with HIZs and MCs. The significantly higher vertebral T2-value for MC Type I and/or with HIZ in adjacent IVDs may be a reflection of a general inflammatory state, since higher T2-values, at least partly, reflect the higher content of water molecules and MC Type I is believed to display edema (9). 
$\mathrm{MC}$ is a common phenomenon for spinal degenerative diseases, but it is not fully elucidated what mechanisms leads to MCs. Abnormal load and stress are hypothesized to affect the EPs and the microenvironment of the adjacent vertebrae, resulting in histological changes that exhibit signal changes on MR images, i.e. MCs. Another hypothesis is that MCs result from an inflammatory reaction by toxic substances from the IVD (22). Also HIZ is assumed to have an inflammatory component originating from proinflammatory substances appearing in the annular tears $(6,23)$. Crock et al. $(24)$ found that upregulation of inflammatory mediators within the IVD could result in local inflammation in the EPs and vertebrae associated with LBP. This finding was confirmed by Rannou et al. (25). Moreover, Ohtori et al. (26) argued that inflammatory mediators and nerve ingrowth into the EPs might be a cause of LBP, and that MC Type I more likely represents earlier changes with presence of proinflammatory chemical mediators whereas MC Type II/III appear to represent more stable changes. Moreover, T2-mapping during aIMRI revealed differences in the loading effect for EPs associated with MCs that might reflect constrained EP-functionality. Also, the strong associations found between HIZ, MC and EP-changes may all reflect impairment in the motion segment. If the simultaneous presence of these MRI findings is linked to a more active ongoing inflammatory process and if this is related to a different pain pattern remains to be investigated.

Numerous studies have been performed with various results linking HIZ with spinal pain. This might be due to differences in the study protocols, e.g. differences in the ROI delineation and in the HIZ definition regarding the signal visibility in the T2-weighted as well as in the T1-weighted images for phenotyping of $\mathrm{HIZ}$ into double and single HIZ (20). The inconsistency found in the literature may also reflect a need for improved HIZ-phenotyping and that HIZ with a presence of associated EP and vertebral changes might represent an additional phenotype. Separation of EP-changes into subtypes, e.g calcifications, erosions and fissures, might also be relevant for the evaluation of spinal pain. In this study, EP-changes were only categorized into existing or non-existing findings using standardized MRI-methods with their intrinsic limitations in contrast and spatial resolution. Nevertheless, association were found. Non-cartesian MRImethods have recently been developed for improved morphological visualization of the EPs (27). With the use of such methods, EP-phenotyping might be feasible.

\section{Limitations}

The small number of LBP-patients and thereby no possibility to relate these MRI findings to pain levels or pattern limits the strength of the conclusion. We, therefore, encourage further larger studies to elucidate the clinical importance of the present findings.

In this study, the EP ROls were positioned over the EP-zone, approximately one pixel from the visible edge of the IVD and vertebra to reduce the influence of these adjacent tissues on the EP T2-value. With use of such strategy, the segmentation does not rely on edges in the image for the delineation and this may 
affect the reproducibility of the T2-value. Nevertheless, the ICC displayed fair agreement between repeated measurements both within and between observers.

\section{Conclusions}

This study of LBP-patients suggests that HIZ is associated with simultaneous presence of both MCs and EP-changes in the same segment. Moreover, T2-mapping was found to objectively reflect MCs associated with HIZs and T2-mapping during aIMRI revealed functional behaviors of the EPs associated with MCs that might reflect impaired EP-functionality. If these three simultaneous morphological features are linked to an active inflammatory process, reflected as a clinical specific pain profile remains to be investigated.

\section{References}

1. Hoy D, March L, Brooks P, Blyth F, Woolf A, Bain C, et al. The global burden of low back pain: estimates from the Global Burden of Disease 2010 study. Ann Rheum Dis. 2014;73(6):968-74.

2. Teraguchi M, Yim R, Cheung JP, Samartzis D. The association of high-intensity zones on MRI and low back pain: a systematic review. Scoliosis Spinal Disord. 2018;13:22.

3. Namboothiri S, Gore S, Veerasekhar G. Treatment of Low Back Pain by Treating the Annular High Intensity Zone (HIZ) Lesions Using Percutaneous Transforaminal Endoscopic Disc Surgery. Int J Spine Surg. 2018;12(3):388-92.

4. Doniselli FM, Zanardo M, Manfre L, Papini GDE, Rovira A, Sardanelli F, et al. A critical appraisal of the quality of low back pain practice guidelines using the AGREE II tool and comparison with previous evaluations: a EuroAIM initiative. Eur Spine J. 2018;27(11):2781-90.

5. Vagaska E, Litavcova A, Srotova I, Vlckova E, Kerkovsky M, Jarkovsky J, et al. Do lumbar magnetic resonance imaging changes predict neuropathic pain in patients with chronic non-specific low back pain? Medicine (Baltimore). 2019;98(17):e15377.

6. Lam KS, Carlin D, Mulholland RC. Lumbar disc high-intensity zone: the value and significance of provocative discography in the determination of the discogenic pain source. Eur Spine $\mathrm{J}$. 2000;9(1):36-41.

7. Carragee EJ, Paragioudakis SJ, Khurana S. 2000 Volvo Award winner in clinical studies: Lumbar high-intensity zone and discography in subjects without low back problems. Spine (Phila Pa 1976). 2000;25(23):2987-92.

8. Mitra D, Cassar-Pullicino VN, McCall IW. Longitudinal study of vertebral type-1 end-plate changes on MR of the lumbar spine. Eur Radiol. 2004;14(9):1574-81.

9. Modic MT. Modic type 1 and type 2 changes. J Neurosurg Spine. 2007;6(2):150-1; discussion 1. 
10. Zhang JF, Wang GL, Zhou ZJ, Fang XQ, Chen S, Fan SW. Expression of Matrix Metalloproteinases, Tissue Inhibitors of Metalloproteinases, and Interleukins in Vertebral Cartilage Endplate. Orthop Surg. 2018;10(4):306-11.

11. Benneker LM, Heini PF, Alini M, Anderson SE, Ito K. 2004 Young Investigator Award Winner: vertebral endplate marrow contact channel occlusions and intervertebral disc degeneration. Spine. 2005;30(2):167-73.

12. Samartzis D, Borthakur A, Belfer I, Bow C, Lotz JC, Wang HQ, et al. Novel diagnostic and prognostic methods for disc degeneration and low back pain. Spine J. 2015;15(9):1919-32.

13. Waldenberg $\mathrm{C}$, Hebelka H, Brisby H, Lagerstrand K, editors. Significant differences in IVD characteristics between low back pain patients and controls associated with HIZ as revealed with quantitative MRI. The International Society for the Study of the Lumbar Spine; 2018; Banff, Canada.

14. Sharma A, Parsons M, Pilgram T. Temporal interactions of degenerative changes in individual components of the lumbar intervertebral discs: a sequential magnetic resonance imaging study in patients less than 40 years of age. Spine (Phila Pa 1976). 2011;36(21):1794-800.

15. Chen C, Jia Z, Han Z, Gu T, Li W, Li H, et al. Quantitative T2 relaxation time and magnetic transfer ratio predict endplate biochemical content of intervertebral disc degeneration in a canine model. BMC musculoskeletal disorders. 2015;16(1):157.

16. Fields AJ, Han M, Krug R, Lotz JC. Cartilaginous End Plates: Quantitative MR Imaging with Very Short Echo Times-Orientation Dependence and Correlation with Biochemical Composition. Radiology. 2014;274(2):482-9.

17. Lagerstrand $\mathrm{K}$, Hebelka $\mathrm{H}$, Brisby $\mathrm{H}$. Low back pain patients and controls display functional differences in endplates and vertebrae measured with T2-mapping. Eur Spine J. 2019;28(2):234-40.

18. Pfirrmann CW, Metzdorf A, Zanetti M, Hodler J, Boos N. Magnetic resonance classification of lumbar intervertebral disc degeneration. Spine (Phila Pa 1976). 2001;26(17):1873-8.

19. Modic MT, Steinberg PM, Ross JS, Masaryk TJ, Carter JR. Degenerative disk disease: assessment of changes in vertebral body marrow with MR imaging. Radiology. 1988;166(1 Pt 1):193-9.

20. Shan Z, Chen H, Liu J, Ren H, Zhang X, Zhao F. Does the high-intensity zone (HIZ) of lumbar Intervertebral discs always represent an annular fissure? Eur Radiol. 2017;27(3):1267-76.

21. Dudli S, Liebenberg E, Magnitsky S, Lu B, Lauricella M, Lotz JC. Modic type 1 change is an autoimmune response that requires a proinflammatory milieu provided by the 'Modic disc'. Spine J. 2018;18(5):831-44.

22. Braithwaite I, White J, Saifuddin A, Renton P, Taylor BA. Vertebral end-plate (Modic) changes on lumbar spine MRI: correlation with pain reproduction at lumbar discography. Eur Spine J. 1998;7(5):363-8.

23. Chen JY, Ding Y, Lv RY, Liu QY, Huang JB, Yang ZH, et al. Correlation between MR imaging and discography with provocative concordant pain in patients with low back pain. Clin J Pain. 2011;27(2):125-30. 
24. Crock HV. Internal disc disruption. A challenge to disc prolapse fifty years on. Spine (Phila Pa 1976). 1986;11(6):650-3.

25. Rannou F, Ouanes W, Boutron I, Lovisi B, Fayad F, Mace Y, et al. High-sensitivity C-reactive protein in chronic low back pain with vertebral end-plate Modic signal changes. Arthritis Rheum. 2007;57(7):1311-5.

26. Ohtori S, Inoue G, Ito T, Koshi T, Ozawa T, Doya H, et al. Tumor necrosis factor-immunoreactive cells and PGP 9.5-immunoreactive nerve fibers in vertebral endplates of patients with discogenic low back Pain and Modic Type 1 or Type 2 changes on MRI. Spine (Phila Pa 1976). 2006;31(9):1026-31.

27. Bae WC, Statum S, Zhang Z, Yamaguchi T, Wolfson T, Gamst AC, et al. Morphology of the Cartilaginous Endplates in Human Intervertebral Disks with Ultrashort Echo Time MR Imaging. Radiology. 2013;266(2):564-74.

\section{Tables}

Table 1. Associations between the investigated morphological features given as absolute and relative numbers

\begin{tabular}{lllllll}
\hline Feature & $\mathrm{HIZ}=0$ & & $\mathrm{HIZ}=1$ & & $\mathrm{MC}=0$ & $\mathrm{MC}=1$ \\
& superior & inferior & superior & inferior & \\
\hline MC & & & & & \\
$=0$ & $98(86 \%)$ & $106(93 \%)$ & $12(57 \%)$ & $15(71 \%)$ & - & - \\
$=1$ & $16(14 \%)$ & $8(7 \%)$ & $9(43 \%)$ & $6(29 \%)$ & - & - \\
EP change & & & & & & \\
$=0$ & $69(61 \%)$ & $84(74 \%)$ & $2(10 \%)$ & $6(29 \%)$ & $181(70 \%)$ & $5(13 \%)$ \\
$=1$ & $45(40 \%)$ & $30(26 \%)$ & $19(91 \%)$ & $15(71 \%)$ & $77(30 \%)$ & $34(87 \%)$ \\
& & & & & & \\
\hline
\end{tabular}

$\mathrm{HIZ}=$ High intensity zone, $\mathrm{MC}=$ Modic change of any type, $\mathrm{EP}=$ endplate. For the categorical variables, $\mathrm{n}$ is presented in absolute numbers and as percentage.

\section{Figures}




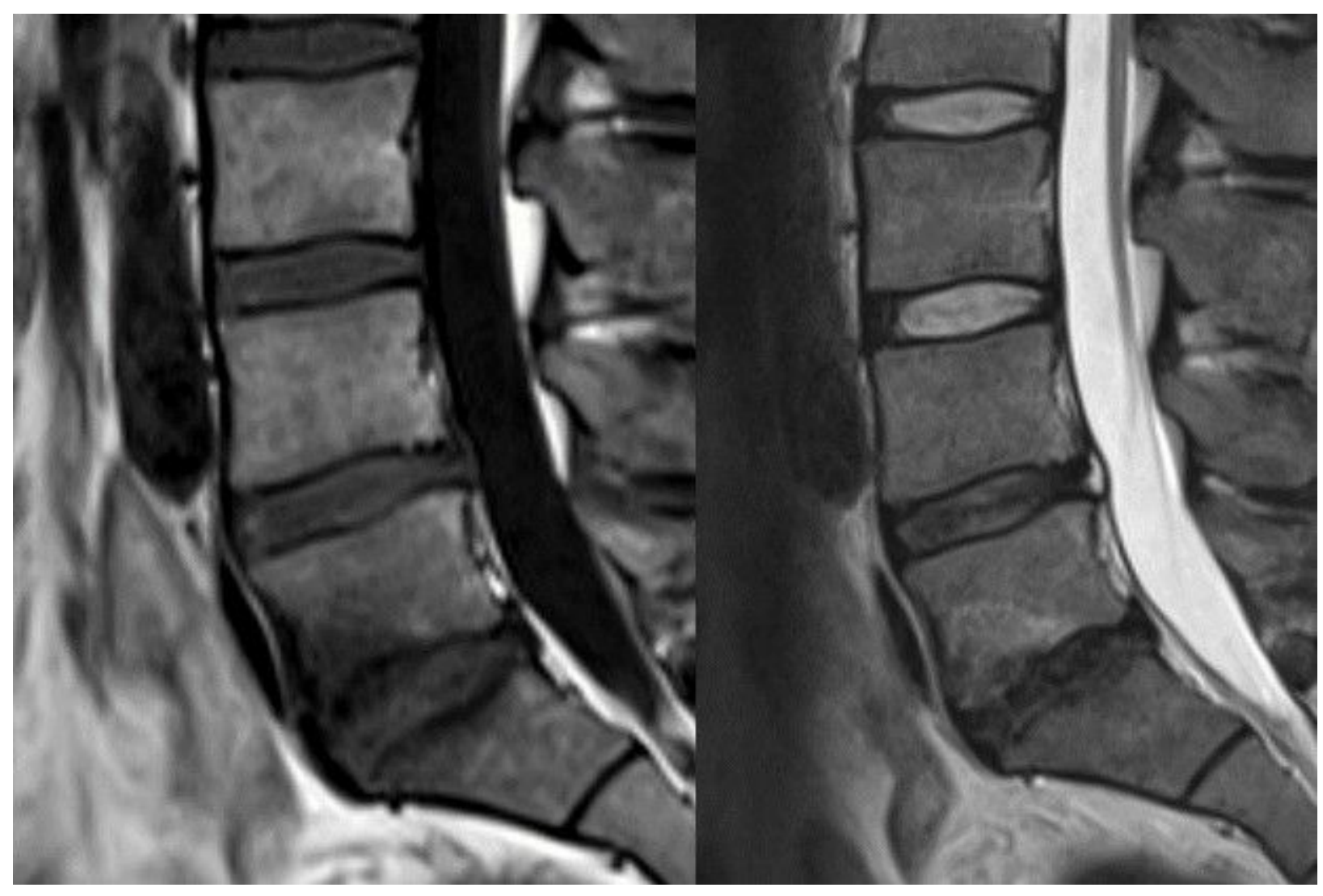

\section{Figure 1}

a) T1W- and b) T2W-images, displaying HIZs, MCs and EP-changes with a wide range of inhomogeneities of the signal in the EP-zone. Modic and discrete EP-changes, as well as HIZ are seen at L4/L5. More extensive EP-changes are seen in EPs adjacent to the L5/S1 IVD. 